\title{
CASE REPORT OPEN Myeloablation-associated deletion of ORF4 in a human coronavirus 229E infection
}

Alexander L. Greninger ${ }^{1,2}$, Gregory Pepper ${ }^{1}$, Ryan C. Shean ${ }^{1}$, Anne Cent ${ }^{1}$, Isabel Palileo ${ }^{1}$, Jane M. Kuypers ${ }^{1}$, Joshua T. Schiffer ${ }^{2,3}$ and $^{1}$ Keith R. Jerome ${ }^{1,2}$

We describe metagenomic next-generation sequencing (mNGS) of a human coronavirus 229E from a patient with AML and persistent upper respiratory symptoms, who underwent hematopoietic cell transplantation (HCT). mNGS revealed a 548-nucleotide deletion, which comprised the near entirety of the ORF4 gene, and no minor allele variants were detected to suggest a mixed infection. As part of her pre-HCT conditioning regimen, the patient received myeloablative treatment with cyclophosphamide and $12 \mathrm{~Gy}$ total body irradiation. Iterative sequencing and RT-PCR confirmation of four respiratory samples over the 4-week peritransplant period revealed that the pre-conditioning strain contained an intact ORF4 gene, while the deletion strain appeared just after conditioning and persisted over a 2.5-week period. This sequence represents one of the largest genomic deletions detected in a human RNA virus and describes large-scale viral mutation associated with myeloablation for HCT.

npj Genomic Medicine (2017)2:30 ; doi:10.1038/s41525-017-0033-4

\section{INTRODUCTION}

Coronaviruses are positive-stranded RNA viruses with large genomes, ranging in size from $25-35 \mathrm{~kb}$. Although much attention has been paid to the epidemic potential and high mortality rates for Middle East Respiratory Syndrome coronavirus and Severe Acute Respiratory Syndrome coronavirus coronaviruses, the most common human coronaviruses (HCoV) include 229E, OC43, HKU1, and NL63 viruses. ${ }^{1}$ HCoV $229 \mathrm{E}$ and NL63 are alphacoronaviruses with genome lengths of $27-28 \mathrm{~kb}$, and HCoV HKU1 and OC43 are betacoronaviruses with genome lengths around $30-31 \mathrm{~kb}$. $^{2}$ Few studies have been conducted on the genome evolution of the common HCoV species. ${ }^{3,4}$

$\mathrm{HCoVs}$ enact a major burden on hematopoietic cell transplant (HCT) patients with $>10 \%$ cumulative incidence of $\mathrm{HCoV}$ infection among HCT by day 100 of transplant. ${ }^{5} \mathrm{HCoV}$ in $\mathrm{HCT}$ patients is associated with prolonged shedding, with a median duration of 3 weeks. ${ }^{5}$ Conditioning regimens for HCT usually consist of chemotherapy and irradiation that may induce genomic alterations. ${ }^{6,7}$ Treatment-related myeloid leukemias are well-known to be associated with radiation therapy or chemotherapeutic agents such as cyclophosphamide. ${ }^{8}$ Here we describe the generation of a large deletion in an RNA virus that was temporally associated with the myeloablative conditioning regimen for HCT.

\section{RESULTS}

The virus analyzed was from a female in her $40 \mathrm{~s}$ with acute myelogeneous leukemia (AML) presenting for hematopoetic cell transplant. The previous year she had undergone four cycles of chemotherapy (G-CLAM, G-CLA, cytarabine, and decitabineprimed MEC). Her prior month's bone marrow evaluation showed $16 \%$ blasts by flow cytometry with normal cytogenetics. On day -12 (12 days prior to transplant), she was found to be infected by a coronavirus 229E with a cycle threshold $(\mathrm{Ct})$ of 30 and persisted at the same Ct on day -9 (Fig. 1a). Based on the patient's risk of relapsing leukemia and relative rarity of $\mathrm{HCoV}$ pneumonitis, the decision was made to continue with HCT despite ongoing viral shedding. On days -7 and -6 she received cyclophosphamide $60 \mathrm{mg} / \mathrm{kg}$, and on days $-4,-3$, and -2 she received a dose of twice-daily $2 \mathrm{~Gy}$ total body irradiation for a cumulative dose of 12 Gy (Fig. 1a). Other significant drugs the patient received during this time period included acyclovir, granisetron, micafungin, voriconazole, and sulfamethoxazole-trimethoprim. A mismatched unrelated donor peripheral blood stem-cell transplant was performed on day 0 . Throughout the $\mathrm{HCoV}$ infection, the patient had mild nasal congestion but never required supplemental oxygen. Her course was complicated by bacteremia on day 10 (10 days after transplant), thought to be due to grade 2 mucositis, and she was discharged on day 20.

Four nasal swab samples over a four-week period were available from the patient. All qRT-PCR cycle thresholds $(\mathrm{Ct})$ recovered over the four-week period were comparable, ranging between 31.3-33.5 (Table 1). The percent of total reads mapping to $\mathrm{HCoV}$ $229 \mathrm{E}$ ranged from $0.01-0.75 \%$, consistent with the low concentrations of virus found by qRT-PCR (Table 1). mNGS of the Day 15 post-transplant specimen revealed a large deletion that encompassed 548 of the 660 nucleotides of the ORF 4 gene (Fig. 1b) with average coverage of $68 \times$ across the viral genome. Repeat library preparations of the same specimen demonstrated the same deletion. The deletion was found whether mapping to the HCoV $229 \mathrm{E}$ reference genome or the de novo assembly. No read was recovered within the area of the deletion, suggesting the presence of a viral population entirely containing the genomic deletion. mNGS of the day -12 and day -9 specimens from the same patient revealed a completely intact ORF4a/4b (Fig. 1b). Other than the large deletion, no consensus variants were observed

\footnotetext{
${ }^{1}$ Department of Laboratory Medicine, University of Washington, Seattle, WA, USA; ${ }^{2}$ Vaccine and Infectious Disease Division, Fred Hutchinson Cancer Research Center, Seattle, WA, USA and ${ }^{3}$ Department of Medicine, University of Washington, Seattle, WA, USA

Correspondence: Alexander L. Greninger (agrening@uw.edu)
}

Received: 10 May 2017 Revised: 28 August 2017 Accepted: 6 September 2017

Published online: 09 October 2017 
A

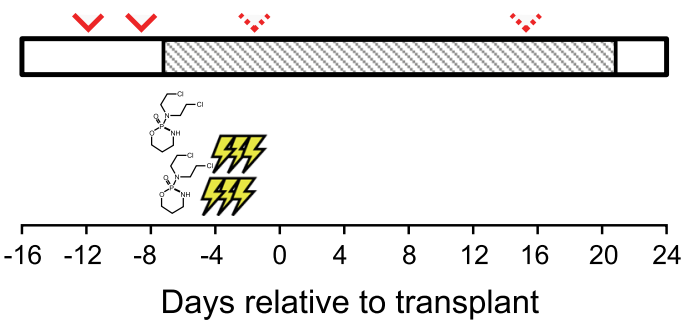

C

$\checkmark$ HCoV 229E intact strain

$\because \quad$ HCoV 229E deletion strain

$\square$ Outpatient oncology

$\mathbf{B}$ Inpatient oncology

52 Gy total body irradiation

Cyclophosphamide

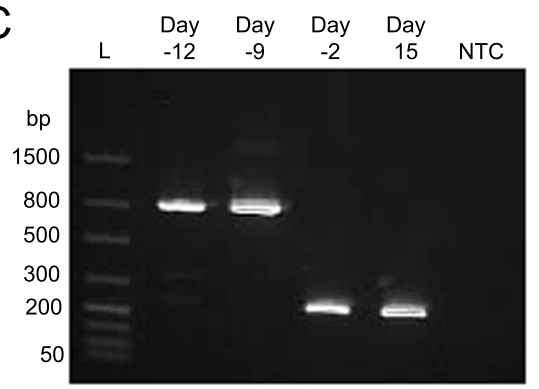

B

Day -12

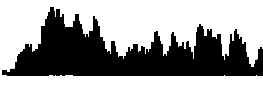

Whinkwh $n+m+n+4$ and

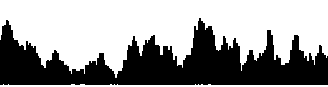
$27 \mathrm{X}$ average Day -9 Monerage

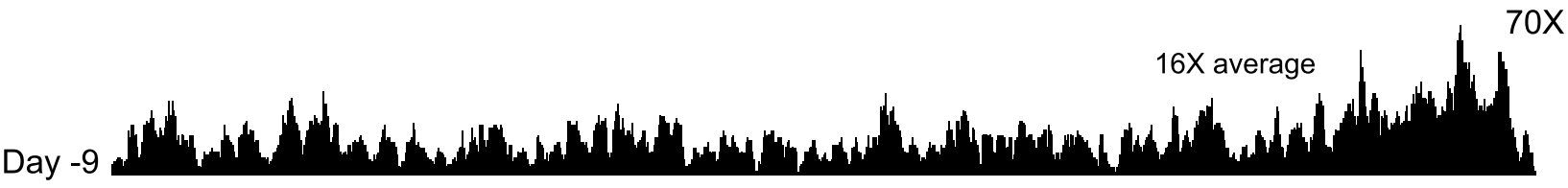

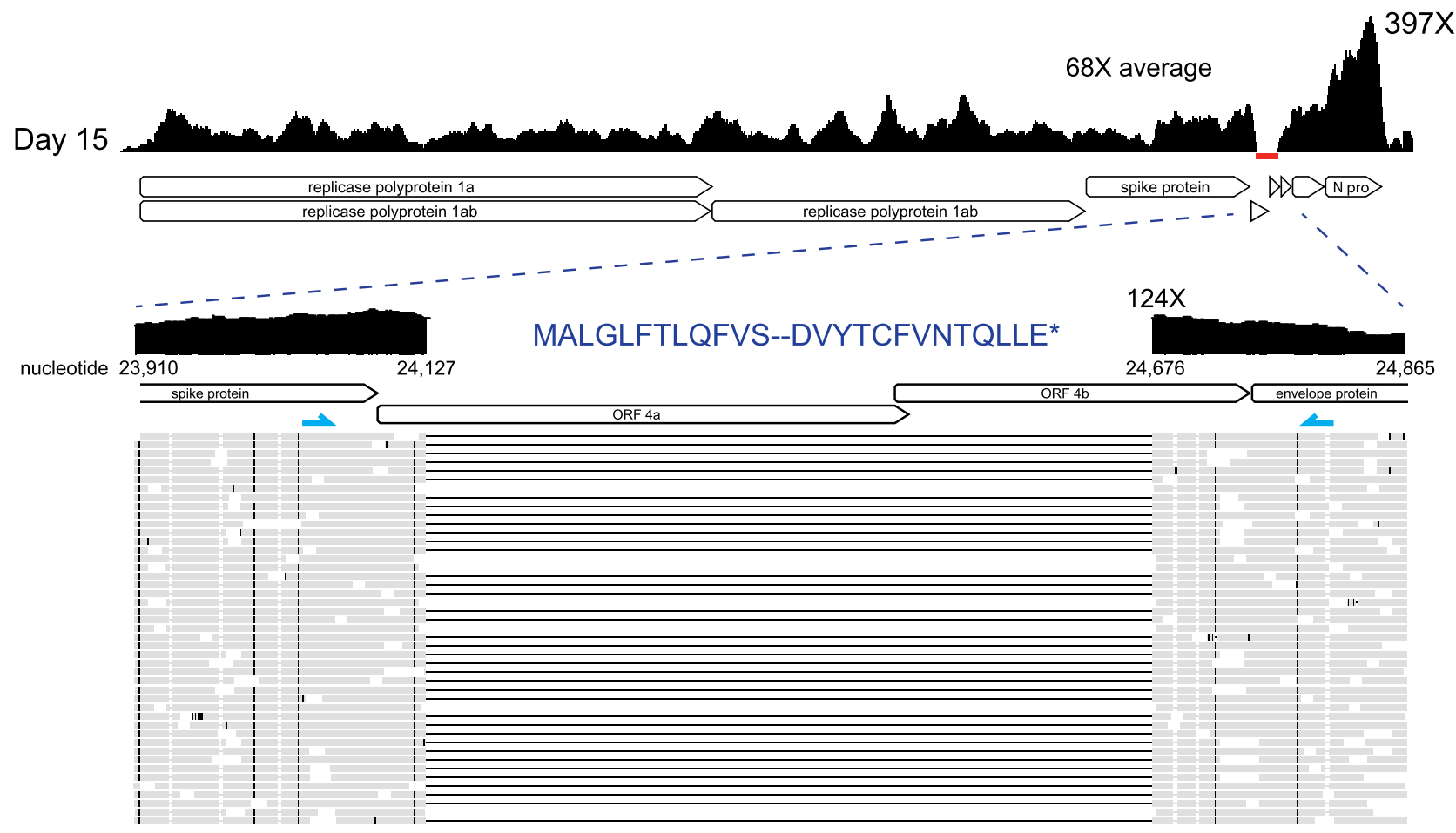

Fig. 1 Detection of a 548-nucleotide deletion in a HCoV 229E strain associated with myeloablation. a Depiction of the case history surrounding myeloablation and coronavirus infection. A woman in her 40s with AML was found to be infected with a HCoV $229 \mathrm{E}$ just before her HCT. Due to the patient's advanced disease, the decision was made to continue with the transplant. Four successive HCoV-positive nasal swab specimens were available from the patient; two from before treatment with myeloablative cyclophosphamide and total body irradiation and two from after. All days are reported relative to HCT. b Coverage plots of mNGS reads mapped to the reference genome for HCoV $229 \mathrm{E}$ (NC_002645) of three samples from the patient revealed the generation of a 548-nucleotide deletion in the ORF4a/4b gene. In addition to deleting $83 \%$ of the coding region from ORF $4 \mathrm{a} / 4 \mathrm{~b}$, the deletion resulted in a frame shift that resulted in a premature stop codon 10 amino acids before the reference genome stop codon. Primer binding sites are denoted in blue for confirmatory junction RT-PCR. c Confirmatory junction RT-PCR of the four available HCoV-positive nasal swabs revealed the absence of the deletion before myeloablation and the presence of the deletion in specimens taken after myeloablation. NTC, no template control

between the HCoV 229E consensus genomes recovered from day -12 , day -9 , and day -15 specimens. Mapping of reads from the day -12 , day -9 , day -15 samples to the day -12 consensus genome revealed two minor allele variants that reached $>10 \%$ allele frequency and had consistent allele frequencies between the day -12 and day -9 samples. The first was a c.12332 A > G, p. $\mathrm{K} 4111 \mathrm{R}$ change in the replicase protein that went from undetectable in the day -12 and day -9 specimens and was at 
Table 1. Specimens sequenced in this study

\begin{tabular}{lllll}
\hline Day & HCoV Ct & HCoV reads & Total reads & Strain name \\
\hline-12 & 31.5 & 7257 & $4,395,609$ & SC379 \\
-9 & 33.5 & 3733 & $30,166,065$ & SC399 \\
-2 & 32.7 & 5064 & $11,185,156$ & SC475 \\
15 & 31.3 & 11,990 & $1,594,894$ & SC677 \\
\hline
\end{tabular}

Note: mNGS sequencing reads to $\mathrm{HCoV} 229 \mathrm{E}$ and total reads for each specimen are indicated, along with qRT-PCR cycle threshold

$11.9 \%$ allele frequency in the day -15 specimen. The second was a c.675_676insU that maintained at $10.6 \%, 10.8 \%$, and $8.9 \%$ allele frequency in the day -12 , day -9 , and day -15 specimens.

No junctional reads across the 548-nucleotide deletion were found in either the day -12 or day -9 specimen by mNGS. mNGS of the day -2 specimen also demonstrated the 548-nucleotide deletion with coverage across the genome consistent with RNA degradation (Figure S1). No consensus variants were observed in the genomic regions covered in the day -2 specimen. Confirmatory RT-PCR across the ORF $4 a / 4 b$ gene demonstrated an intact gene in the pre-myeloablation specimens and a smaller PCR product consistent with the 548-nucleotide deletion in the day -2 and day 15 post-myeloablation specimens (Fig. 1c). Deep sequencing of the day -9 and day -2 PCR amplicons revealed no junction reads from the day -9 specimen mapping to the day 15 deletion strain locus with $11,279 \times$ coverage at the same locus for the intact ORF4 gene in the day -12 strain (Figure S2). Conversely, only one paired-end read from the day -2 specimen mapped to the deleted portion of the ORF4 gene locus in the day -12 strain with $158,938 \times$ coverage at the ORF4 locus in the day -15 deletion strain (Figure S2).

Attempts to culture each of the patient's specimens on MRC-5 cells proved unsuccessful. No CPE was visualized for any of the patient's four clinical samples and HCoV 229E RNA levels in the culture supernatant were undetectable by qRT-PCR after inoculation, while viral growth was apparent in the positive control HCoV 229E ATCC type strain (Figure S3).

\section{DISCUSSION}

We describe metagenomic detection of a large deletion in a $\mathrm{HCoV}$ $229 \mathrm{E}$ strain temporally associated with a myeloablative regimen of cyclophosphamide and total body irradiation in an HCT patient. The ORF4-deleted virus maintained the same viral load over a 2.5 week period and was clonal by day 15 after transplant, suggesting that it was replication competent in vivo. No other majority consensus variants in the $\mathrm{HCoV}$ genome arose during the sampled period. One minor variant arose in the day 15 specimen that was not present in the earlier specimens, while another minor variant maintained a similar allele frequency throughout the sampled period. This rate of single nucleotide variant evolution is consistent with the molecular clock of $\mathrm{HCoV}$ and with a study of that found no HCoV genomic variants arose during the first month of infection. ${ }^{9}$ The ability of the virus to persist in vivo despite the near complete loss of ORF4 suggests that this accessory gene is completely dispensable for HCoV $229 \mathrm{E}$ replication and may outcompete the wild-type virus under certain conditions. Indeed, HCoV 229E type strain contains a 2-nucleotide deletion that splits ORF4 into ORF4a/4b, and the alpaca alphacoronavirus contains a 1 -nucleotide insertion that also disrupts the reading frame. ${ }^{10,11}$ Meanwhile, all other group $1 \mathrm{~b}$ coronaviruses including all known HCoV 229E clinical strains contain an intact ORF4. ${ }^{10,12}$ This is the first detection of a non-intact ORF4 in HCoV 229E associated with human infection.
The function of ORF4 in the alphacoronaviruses is unknown. The protein sequence shows no significant alignment to any protein in Protein Data Bank by HHPred. ${ }^{13}$ ORF4a of the HCoV 229E type strain has been suggested to be a viroporin that possessed ion channel activity. ${ }^{14}$ Interestingly, replacement of ORF4 in the HCoV 229 E type strain with Renilla luciferase resulted in viral replication in vitro comparable to that of the wild-type strain, consistent with our in vivo data that ORF4 is unlikely to be required for replication. ${ }^{15}$ Taken together, these data suggest that ORF4 would make a poor antiviral target for the human coronaviruses.

Many potential mechanisms for development of the deletion exist. The chemotherapy and/or irradiation could have been directly toxic to the coronavirus genome. Cyclophosphamide is a well-known clastogen and has recently been associated with increased development of single nucleotide variations insertions and deletions. ${ }^{7,16}$ Alternatively, the myeloablative regime could have removed immune pressure, allowing for the loss of ORF4. It seems that in certain circumstances, deletion of ORF4 could prove a selective advantage as it was the only allele present in the day -15 specimen.

This study has several limitations. We were unable to culture any of the patient's HCoV specimens to demonstrate ability to replicate in vitro. This is not necessarily surprising as HCoV $229 \mathrm{E}$ are not usually cultured in the clinical virology lab and the virus was present at low concentrations in the sample. We are also limited in accurately calling minor variants by the relatively low coverage of each viral genome. We did not attempt reverse genetics of any alphacoronaviruses to definitively show ORF4 is dispensable for in vitro replication, although the ability of the type strain to replicate despite a 2-nucleotide deletion in ORF4 and the luciferase gene replacement experiment described above are consistent with this possibility.

In summary, we describe a large deletion in HCoV $229 \mathrm{E}$ encompassing almost an entire gene that was temporally associated with myeloblation. Little is known about the genome evolution of HCoV $229 \mathrm{E}$ as only 20 genomes are currently available in NCBI Genbank. Similarly sized (500-700 nucleotide) deletions have previously been found in hepatitis $D$ virus in patients receiving antiviral therapy. ${ }^{17}$ Our study is also the first demonstration of microbial evolution associated with cyclophosphamide treatment or irradiation. Further research into how chemotherapy and irradiation affect the microbiome is warranted. As mNGS is increasingly performed in the clinical lab, ${ }^{18}$ much intriguing biology can be unlocked from clinical specimens, providing a continuing case for the sequencing clinical microbial genomes. ${ }^{19-21}$

\section{MATERIALS AND METHODS}

Cell culture and qRT-PCR

The HCoV 229E type strain ATCC VR-740 was obtained and used as a positive control for cell culture. Two hundred microliter of each patient nasal swab in viral transport media $(50 \mu \mathrm{L} 229 \mathrm{E}$ control/150 $\mu \mathrm{L} 2 \% \mathrm{MEM})$ was inoculated onto MRC-5 cells (ATCC CCL-171) and incubated at $37^{\circ} \mathrm{C}$ in stationary rack for $30 \mathrm{~min}$. After incubation, $1.2 \mathrm{~mL}$ of $2 \%$ MEM was added to each tube and incubated at $37^{\circ} \mathrm{C}$. Each day tubes were read for cytopathic effect and $50 \mu \mathrm{L}$ of supernatant was stored at $-80^{\circ} \mathrm{C} .50 \mu \mathrm{L}$ culture supernatants were extracted on a Roche MagNA Pure LC 2.0 and RNA was eluted in $100 \mu \mathrm{L}$ water. Ten microliter of RNA was used for qRTPCR with primers targeting the HCoV polymerase gene. ${ }^{22}$ qRT-PCR on patient samples was performed on $200 \mu \mathrm{L}$ of nasal swab in viral transport with the same extraction and PCR conditions.

Metagenomic next-generation sequencing and confirmatory RTPCR with deep sequencing

mNGS was performed as described previously. ${ }^{23}$ Briefly, $20 \mu \mathrm{L}$ of extracted RNA was treated with DNAse I and double stranded CDNA was created using random hexamers with SuperScript III and Sequenase v2.0 (Thermo Fisher). mNGS libraries were created using one-third volume NexteraXT reactions with 16 cycles of PCR amplification. mNGS libraries from the 
deletion-containing specimens were prepared twice from RNA. Dualindexed libraries were sequenced on an Illumina MiSeq over several different types of runs $(1 \times 190 \mathrm{bp}, 1 \times 188 \mathrm{bp}, 2 \times 94 \mathrm{bp}, 2 \times 300 \mathrm{bp})$ with consistent results. Sequencing data were Nextera adapter and quality (Q30) trimmed using cutadapt, iteratively mapped to the HCoV $229 \mathrm{E}$ reference genome (NC 002645) using the Geneious v9.1 read aligner, de novo assembled using SPAdes v3.9, and visualized using Geneious v9.1 and manually curated. ${ }^{24-26}$ The HCoV $229 \mathrm{E}$ reference genome and the strains sequenced in this study have an average pairwise nucleotide identity of $98 \%$. Majority consensus genomes from mNGS were called using a minimum of $3 \times$ coverage. Reads for each specimen were also mapped to the day -12 consensus genome to look for minor variants that arose during treatment. Due to low coverage, minor variants were called with a minimum allele frequency of $10 \%$ at $11 \times$ coverage, after trimming of inversions due to Nextera artifacts, variants within 10 nucleotides of end of read, and PCR duplicates.

Confirmatory gap junction RT-PCR was performed on $1 \mu \mathrm{L}$ of RNA extracted as above using 35 cycles following manufacturer's recommended conditions (Tm 55 C) using quarter-volumes of the Qiagen OneStep RT-PCR kit and primers HCoV_24034F (5'-TTGTTGTGAATCAACTAAACTTCC-3') and HCoV_24808R (5'-ACACACCAGAGTAGTACATTAAC $\left.-3^{\prime}\right)$. Deep sequencing of the day -9 and day -2 PCR amplicons was performed using $1 \mathrm{ng}$ of PCR product purified with the Zymo DNA Clean and Concentrator Kit-5 with 1/10th volumes of the the Kapa HyperPlus kit ligation of Y-shaped adapters followed by seven cycles of Truseq-adapter dual-indexed PCR amplification. ${ }^{27}$ Sequencing was performed using a $315 \times 310$ bp run on an Illumina MiSeq. From the day -9 amplicon 36,880 paired-end reads were recovered, while from the day -2 amplicon 231,990 paired-end reads were recovered. PCR products were Truseq-adapter and quality-trimmed using bbduk and aligned to the ORF4 intact and deleted alleles present in the day -12 and day -15 consensus genomes using the Geneious read aligner with no gaps or mismatches allowed. ${ }^{28}$

The information disclosed in this case report complies with HIPAA Privacy Rule requirements about Protected Health Information. The patient provided written informed IRB-approved consent for research on excess clinical specimens.

\section{Data availability statement}

These sequences are deposited in NCBI Genbank under the strain names SC677 day 15 (KY369909), SC379 day -12 (KY621348), and SC399 day -9 (KY674914). The non-human reads are deposited in the NCBI Short Read Archive under accessions SRR5809357-SRR5809372, SRR5809395, SRR5816371-SRR5816372.

\section{ACKNOWLEDGEMENTS}

The authors acknowledge Dr. Michael Boeckh and Terry Stevens-Ayers for assistance with patient consent.

\section{AUTHOR CONTRIBUTIONS}

A.L.G., G.P., I.P. performed experiments; A.L.G., R.C.S., A.C., J.M.K. analyzed data; A.L.G., J.T.S., K.R.J. wrote the paper.

\section{ADDITIONAL INFORMATION}

Supplementary information accompanies the paper on the npj Genomic Medicine website (https://doi.org/10.1038/s41525-017-0033-4).

Competing interest: The authors declare that they have no competing financial interests.

Publisher's note: Springer Nature remains neutral with regard to jurisdictional claims in published maps and institutional affiliations.

\section{REFERENCES}

1. Lepiller, Q. et al. High incidence but low burden of coronaviruses and preferential associations between respiratory viruses. J. Clin. Microbiol. 51, 3039-3046 (2013).

2. Forni, D., Cagliani, R., Clerici, M. \& Sironi, M. Molecular evolution of human coronavirus genomes. Trends Microbiol. 25, 35-48 (2017).

3. Dijkman, R. \& van der Hoek, L. Human coronaviruses 229E and NL63: Close yet still so far. J. Formos. Med. Assoc. 108, 270-279 (2009).
4. Posthuma, C. C., Te Welthuis, A. J. W. \& Snijder, E. J. Nidovirus RNA polymerases: complex enzymes handling exceptional RNA genomes. Virus Res. https://doi.org/ 10.1016/j.virusres.2017.01.023 (2017).

5. Milano, F. et al. Human rhinovirus and coronavirus detection among allogeneic hematopoietic stem cell transplantation recipients. Blood 115, 2088-2094 (2010).

6. Gyurkocza, B. \& Sandmaier, B. M. Conditioning regimens for hematopoietic cell transplantation: one size does not fit all. Blood 124, 344-353 (2014).

7. Szikriszt, B. et al. A comprehensive survey of the mutagenic impact of common cancer cytotoxics. Genome Biol. 17, 99 (2016).

8. Godley, L. A. \& Larson, R. A. Therapy-related Myeloid Leukemia. Semin. Oncol. 35, 418-429 (2008).

9. Ogimi, C. et al. Prolonged shedding of human coronavirus in hematopoietic cell transplant recipients: risk factors and viral genome evolution. J. Infect. Dis. https:// doi.org/10.1093/infdis/jix264.

10. Dijkman, R. et al. Human coronavirus 229E encodes a single ORF4 protein between the spike and the envelope genes. Virol. J. 3, 106 (2006).

11. Crossley, B. M., Mock, R. E., Callison, S. A. \& Hietala, S. K. Identification and characterization of a novel alpaca respiratory coronavirus most closely related to the human coronavirus 229E. Viruses 4, 3689-3700 (2012).

12. Farsani, S. M. J. et al. The first complete genome sequences of clinical isolates of human coronavirus 229E. Virus Genes 45, 433-439 (2012).

13. Söding, J., Biegert, A. \& Lupas, A. N. The HHpred interactive server for protein homology detection and structure prediction. Nucleic Acids Res. 33, W244-W248 (2005).

14. Zhang, R. et al. The ORF4a protein of human coronavirus $229 \mathrm{E}$ functions as a viroporin that regulates viral production. Biochim. Biophys. Acta BBA-Biomembr. 1838, 1088-1095, (2014).

15. Worm, S. H. Evanden et al. Reverse genetics of SARS-related coronavirus using vaccinia virus-based recombination. PLOS ONE 7, e32857 (2012).

16. Povirk, L. F. \& Shuker, D. E. DNA damage and mutagenesis induced by nitrogen mustards. Mutat. Res. 318, 205-226 (1994).

17. Hsu, C.-W. et al. Detection of hepatitis D virus RNA carrying large fragment deletions in patients with severe hepatitis $B / D$ receiving oral antiviral therapy. J. Med. Virol. 87, 634-641 (2015).

18. Greninger, A. L. et al. A metagenomic analysis of pandemic influenza A (2009 H1N1) infection in patients from North America. PLOS ONE 5, e13381 (2010).

19. Greninger, A. L. et al. Copy number heterogeneity of JC virus standards. J. Clin. Microbiol. https://doi.org/10.1128/JCM.02337-16 (2016).

20. Greninger, A. L. et al. Clinical metagenomic identification of Balamuthia mandrillaris encephalitis and assembly of the draft genome: the continuing case for reference genome sequencing. Genome Med. 7, 113 (2015).

21. Canuti, M. \& van der Hoek, L. Virus discovery: are we scientists or genome collectors? Trends Microbiol. 22, 229-231 (2014).

22. Kuypers, J. et al. Clinical disease in children associated with newly described coronavirus subtypes. Pediatrics 119, e70-e76 (2007).

23. Greninger, A. L. et al. Rapid metagenomic next-generation sequencing during an investigation of hospital-acquired human parainfluenza virus 3 infections. J. Clin. Microbiol. 55, 177-182 (2017).

24. Martin, M. Cutadapt removes adapter sequences from high-throughput sequencing reads. EMBnet J. 17, 10-12 (2011).

25. Kearse, M. et al. Geneious basic: an integrated and extendable desktop software platform for the organization and analysis of sequence data. Bioinformatics 28, 1647-1649 (2012).

26. Bankevich, A. et al. SPAdes: A new genome assembly algorithm and its applications to single-cell sequencing. J. Comput. Biol. 19, 455-477 (2012).

27. Koelle, D. M. et al. Worldwide circulation of HSV-2XHSV-1 recombinant strains. Sci. Rep. 7, 44084 (2017)

28. BBTools. DOE Joint Genome Institute. http://jgi.doe.gov/data-and-tools/bbtools/. Accessed: 11 July 2017.

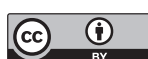

Open Access This article is licensed under a Creative Commons Attribution 4.0 International License, which permits use, sharing, adaptation, distribution and reproduction in any medium or format, as long as you give appropriate credit to the original author(s) and the source, provide a link to the Creative Commons license, and indicate if changes were made. The images or other third party material in this article are included in the article's Creative Commons license, unless indicated otherwise in a credit line to the material. If material is not included in the article's Creative Commons license and your intended use is not permitted by statutory regulation or exceeds the permitted use, you will need to obtain permission directly from the copyright holder. To view a copy of this license, visit http://creativecommons. org/licenses/by/4.0/.

(c) The Author(s) 2017 